\title{
Calidad y desarrollo en la educación a distancia
}

\section{Quality and development in distance education}

Econ. Lucía Pico, Mgs.

Universidad Internacional del Ecuador, Ecuador

Autor para correspondencia: lucia_pico@ hotmail.com

Fecha de recepción: 17 de Marzo de 2016 - Fecha de aceptación: 20 de Mayo de 2016

\section{Resumen}

La Educación a distancia se encuentra en creciente desarrollo y esto se evidencia a través de la variedad de programas que ofrecen las instituciones públicas y privadas en Latinoamérica y el mundo entero. A eso se suma la incorporación de las tecnologías de la información y comunicación, herramienta útil para viabilizar estos programas en la era de globalización en que nos encontramos inmersos. En el Ecuador, está tomando gran importancia el realizar la evaluación de la calidad en programas de educación superior, partiendo de los conceptos básicos y normativas de gran relevancia emitidas por los organismos reguladores de la evaluación y acreditación de la calidad.

Palabras claves: educación superior; educación a distancia; calidad

\begin{abstract}
Distance education is increasingly developing and this is evidenced by the variety of programs offered by public and private institutions in Latin America and the world. Hence the incorporation of information technologies and communication, useful for these programs viable in the era of globalization in which we are immersed tool adds. In Ecuador, it is taking great importance to the evaluation of quality in higher education programs, starting from the basics and highly relevant regulations issued by regulatory agencies of the evaluation and accreditation of quality.
\end{abstract}

Key words: higher education; distance education; quality 


\section{Introducción}

La misión de la educación superior es formar ciudadanos y profesionales con las cualidades necesarias para la construcción de sociedades democráticas y desarrolladas (Días Sobrinho, Goergen, 2006). Se considera pertinente resaltar la gran contribución hacia avances económicos, rescatando la pluralidad de los distintos grupos sociales.

Ofertar programas de calidad en educación superior se torna progresivo e importante, y nos invita a reflexionar en la pertinencia de la planificación, diseño y construcción de los programas de educación a distancia, que vayan acorde a la realidad y exigencias actuales. Parte de este cambio lo compone la cultura, recordar que no solo depende de las instituciones sino también de una cultura que incluya la participación de cada uno como actores principales considerando entre ellos docentes, estudiantes, materiales educativos y requerimientos tecnológicos. (García Aretio, 1993).

Se resalta la consideración expresada sobre evaluación de García Llamas (1986) como "el proceso de identificación, recolección y tratamiento de datos para obtener una información que justifique una determinada decisión. Debe servir, no solo para analizar un determinado programa, sino también para ayudarnos a comprender el proceso de aprendizaje"; en este sentido resulta importante que la evaluación sea no solo un proceso de la institución de educación superior sino también de cada docente, profesor o tutor que pueda realizar desde su programa, aula física o virtual; un análisis sólido y científico que permita identificar los puntos en que deba reforzar, profundizar o mejorar en búsqueda de alcanzar calidad.

En la actualidad, el esfuerzo de los organismo de control, han cumplido su cometido con la expedición de la propia reglamentación para las IES que realizan esta oferta educativa, y con ello, se visualiza que en breve, la evaluación de carreras y programas en las modalidades en línea y a distancia, sea una realidad en el país; no obstante esta visión futurista en el ámbito de la evaluación mencionada, fortalece el entusiasmo por contar con una orientación consensuada sobre criterios y subcriterios de evaluación que permitan una evaluación adecuada a los programas de educación. De allí la necesidad de la optimización del talento humano, que sirva como semillero para expandir esta necesidad tan valiosa y requerida en la evaluación de la calidad. El sistema de educación superior tiene como finalidad la formación académica y profesional con visión científica y humanista; la investigación científica y tecnológica; la innovación, promoción, desarrollo y difusión de los saberes y las culturas; la construcción de soluciones para los problemas del país, en relación con los objetivos del régimen de desarrollo. (Constitución de la República del Ecuador Asamblea Constituyente, 2008).

En el Ecuador, el organismo encargado de evaluación y acreditación es el Consejo de Evaluación, Acreditación y Aseguramiento de la Calidad de la Educación Superior (CEAACES) que expidió el Reglamento para Carreras y Programas Académicos en Modalidades en Línea, a Distancia y Semipresencial o de Convergencia de Medios RPC-SE-14-No.043-2015; en el cuál resalta lo siguiente:

Es importante destacar que este objetivo busca formar profesionales acordes a las exigencias del mundo actual, en donde se evidencia la necesidad de generar desarrollo en el sector educativo que sin duda tiene gran relación con los sectores sociales, productivos y 
culturales de cada nación, tomando conciencia que hay relación directa entre la función ejecutiva y la sociedad ecuatoriana, es decir a mayor cantidad de profesionales desarrollados en un país mayor desarrollo del país. Considerando también la disposición tercera del artículo 169 del Reglamento de carrera del CES en el que indica "La oferta y ejecución de programas de educación superior es atribución exclusiva de las instituciones de educación superior legalmente autorizadas" es pertinente enfatizar en que la legalidad de las mismas es la que lleva a estas instituciones a poder ejecutar programas, siendo esta legalidad debidamente autorizada por el Estado durante las jornadas de evaluación y acreditación. En el Ecuador la oferta de programas de educación superior en la modalidad a distancia comenzó hace un poco más de 35 años, por lo que ya hay un camino recorrido en el mismo, y para esto se torna necesario analizar la definición de la modalidad a distancia que indica:

La modalidad a distancia es aquella en la cual, el componente de docencia, el de prácticas de aplicación y experimentación de los aprendizajes y el de aprendizaje autónomo, están mediados por el uso de tecnologías y entornos virtuales bajo plataformas de interacción, y por la articulación de múltiples recursos didácticos (físicos y digitales). Para su desarrollo, es fundamental la labor docente y técnico docente con la tutoría sincrónica y asincrónica, y el respaldo administrativo-organizativo de centros de apoyo. (Reglamento para carreras y programas académicos en modalidad en línea, distancia y semipresencial o de convergencia de medios, CEAACES 2015)

Evaluar esta modalidad de estudio indica la necesidad de crear comunidades académicas que permitan contar con profesionales, los cuales en un espacio de análisis y discusión, realicen las autoevaluaciones pertinentes al sistema que permitan enriquecer el proceso de aprendizaje en todos sus ámbitos. De esta forma contarán con una preparación fundamental para el momento de evaluación y acreditación. El equipo técnico, el personal académico, los coordinadores de centros de apoyo y expertos en tecnología educativa formarán sin duda un gran equipo para viabilizar el proceso de enseñanza aprendizaje acorde a las necesidades actuales. Las funciones del Consejo de Evaluación, Acreditación y Aseguramiento de la Calidad de la Educación Superior son primordiales desde el aspecto de la planificación, coordinación y ejecución de las actividades a realizar para el correcto proceso de evaluación y acreditación, hasta para aprobar la normativa que deben seguir para los mencionados procesos, puesto que se trata de una relación ganar-ganar en donde las instituciones de educación superior debe poner todos sus esfuerzos por estar al máximo nivel y los organismos encargados deben colaborar para que así sea; solo trabajando en conjunto se lograrán que los esfuerzos en conjunto resalten a educación de calidad y contribuyan al desarrollo del país.

Sin duda, no podemos apartar del análisis el gran avance logrado en infraestructura tecnológica por medio de la incorporación de las tecnologías de la Información y comunicación (TICs), las mismas se han convertido en un elemento fundamental dentro de la educación a distancia y en el Ecuador desde el año 2009 se expidió precisamente el Reglamento especial para los programas de educación con el apoyo de las TICs (Resolución del CONESUP RCP.S11.No.260.09 del 3 de septiembre del 2009).

Resulta pertinente realizar también énfasis en la evaluación del aprendizaje, siendo esta una fase que permite obtener información cualitativa y cuantitativa sobre el cumplimiento de los 
objetivos a desarrollar con los estudiantes, en donde cada institución de educación superior determinará las valoraciones de las diferentes actividades académicas (Art. 36 Leyes, Normas y Reglamentos que regulan la Educación Superior a distancia y en línea en América Latina y el Caribe, 2011)

\section{Conclusiones}

Es de gran importancia que existan mecanismos que aseguren la calidad en la educación, desde el momento en que ofertan los programas inclusive cuando los usuarios se encuentren ya en su ejercicio profesional. La práctica evaluativa sigue siendo un desafío al que nos enfrentamos aun con ciertos desaciertos pues requiere de mucha responsabilidad, organización y concienciar el concepto de calidad que necesitamos manejar para que la evaluación de cada acción que se realice dentro del proceso de enseñanza - aprendizaje sea eficaz, crítico y en búsqueda de encontrar la excelencia que nos mantenga aptos para los desafíos que nos exigen los actuales sistemas de evaluación. Las percepciones de los estudiantes también son importantes de considerar pues responden al enriquecimiento de formas y métodos que tenemos para poder llegar con un aprendizaje eficaz bajo los parámetros de calidad en educación superior. El profesorado debe realizar continuamente procesos de autoevaluación que lo lleven a reflexionar en el ejercicio crítico y objetivo del proceso de enseñanza - aprendizaje, acorde a las necesidades e importancia las tecnologías de información y comunicación, vitales para el proceso de globalización que vivimos. Finalmente, se evidencia la necesidad de diseñar y ejecutar modelos de evaluación de calidad en programas de pregrado, siendo que la educación superior es de vital importancia y necesidad en el mundo actual, en donde los profesionales buscan incrementar conocimiento y perfeccionar habilidades que los lleven a ser competitivos en el mundo actual.

\section{Bibliografía}

Barceló H., Padilla A., Santander W., Schalk A.,... Peré N., (2014). Leyes, Normas y Reglamentos que regulan la Educación Superior a Distancia y en Línea en América Latina y el Caribe. II Edición. Disponible en:

Días Sobrinho, J. (2008). Calidad, pertinencia y responsabilidad social de la Universidad latinoamericana y caribeña. http://www.oei.es/salactsi/Capitulo_03_Dias_Sobrinho.pdf

Espinoza, O. (2010). Los sistemas de aseguramiento de la calidad en la Educación Superior en América Latina. $\quad$ http://www.revistaakademeia.cl/wp/wp-content/uploads/2010/08/Los-sistemas-de aseguramiento-de-la-calidad-en-la-educacion-superior-en-America-Latina.pdf

García Llamas, J.L. (s/f). Evaluación de programas educativos: modelos, técnicas e instrumentos, análisis de datos y elaboración de informes. http://www.redescepalcala.org/inspector/Documentos\%20y\%20libros/Evaluacion/Evaluacion\%20y\%20calidad\%2 0\%28uned\%29/Uned_6/webdoc6.htm

$\begin{array}{llllll}\text { Hernández } & \text { Y. } & \text { y } & \text { Ornes } & \text { C. } & \text { (2014). }\end{array}$ http://servicio.bc.uc.edu.ve/educacion/eduweb/v8n2/art05.pdfhttp://www.caledead.org/sites/default/files/files/leyes_normas_reglamentos_ead_2da_ed.pdf Reglamento para carreras y programas académicos en modalidades en línea, a distancia y semipresencial o de convergencia de medios. Consejo de Educación Superior, 16 de diciembre del 2015.

Santángelo H., Alvaro P., Daza R., Roesler J., Vianney J.,....Vargas Ma.Lourdes., (2011).Leyes, Normas y Reglamentos que regulan la Educación Superior a Distancia y en Línea en América Latina y el Caribe. I Edición. http://www.caled-ead.org/sites/default/files/files/leyesnormas.pdf 